\title{
Homeobox Gene
}

National Cancer Institute

\section{Source}

National Cancer Institute. Homeobox Gene. NCI Thesaurus. Code C16615.

A family of genes that contain a highly conserved sequence of approximately 180 nucleotides, which encode an approximately 60 amino acid helix-turn-helix DNA binding domain made up of 2 antiparallel alpha helices and one nearly perpendicular alpha helix connected by short chains of unstructured amino acid sequences. This gene family is involved in transcriptional regulation during embryonic development. 